\title{
Hybridization of the Artificial Immune Network Using the Backpropagation Neural Network
}

\section{Omar Saber Qasim}

omar.saber@uomosul.edu.iq

\author{
Israa Rustum Mohammed \\ israaru.alkhayyat@gmail.com
}

College of Computer Science and Mathematics

University of Mosul, Mosul, Iraq

Received on: 27/09/2012

Accepted on: 03/04/2013

\begin{abstract}
In this research building style simulation developed is applied in the field of pattern recognition medical patients osteoporosis through a process of integrating and hybridization between artificial immune network and back propagation neural network, where the focus was on the qualities positive and overcome the negative qualities possessed by each of these two technologies by building technology improved, have proven technical hybrid it with better results and high efficiency in the classification of cases patients osteoporosis compared with both artificial immune network (AIN) and back propagation neural network (BP).
\end{abstract}

Keywords: artificial neural network; artificial immune network; pattern recognition.

$$
\begin{aligned}
& \text { تهجين الثبكة المناعية الاصطناعية باستخدام شبكة إنتشار الخطأ خلفاً }
\end{aligned}
$$

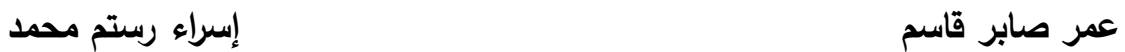

$$
\begin{aligned}
& \text { قسم الرباضيات } \\
& \text { كلية علوم الحاسوب والرياضيات } \\
& \text { جامعة الدوصل، الدوصل، العراق }
\end{aligned}
$$

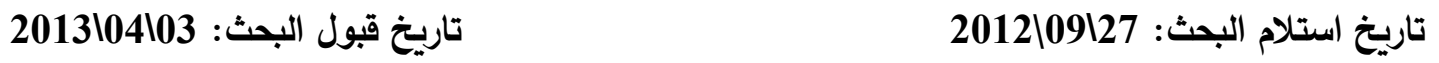

$$
\begin{aligned}
& \text { الملخص }
\end{aligned}
$$

تم في هذا البحث بناء أســلوب محاكاة متطور يتم تطبيقه في مجال التعرف على الأنماط الطبية لمرضــى

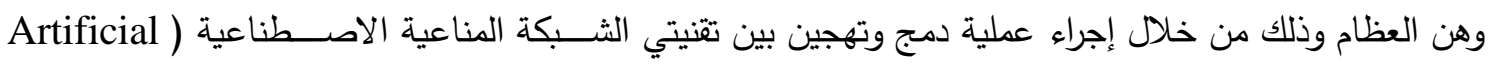
(Immune Network وشـبكة انتشـار الخطأ خلفا (Error Back Propagation Neural Network), إذ تم التركيز على الصـفات الايجابية والتغلب على الصـفات السـلبية التي تمتلكها كل من هاتين التقتيتين من خلال بناء

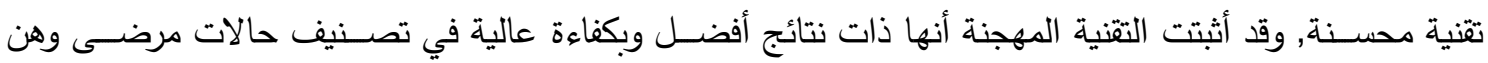
العظام مقارنة مع كل من تقنيتي الثبكة المناعية الاصطناعية (AIN) وشبكة انتشار الخطأ خلفا (BP). الكلمات المفتاحية: الثبكة العصبية الاصطناعية; الثبكة المناعية الاصطناعية; تمييز الأنماط.

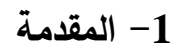

إن التقدم العلمي الذي تشهده العلوم الحديثة المختلفة في العديد من المجالات والتطبيقات وخصوصاً في مجال

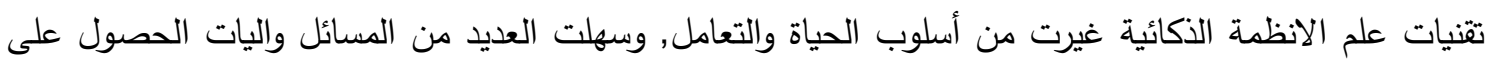


المعلومات وتحليلها, وبالأخص في المجالات الطبية, إذ كان لها الأثر الواضح في حل العديد من المشكلات منها

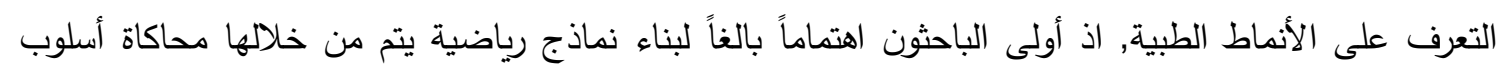

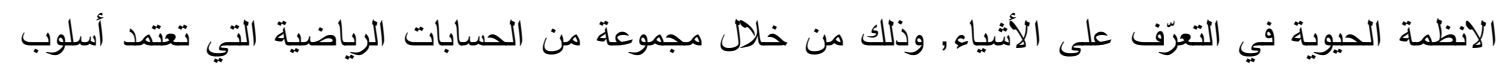

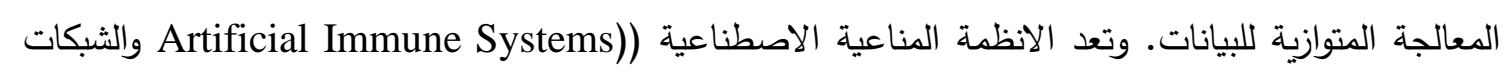

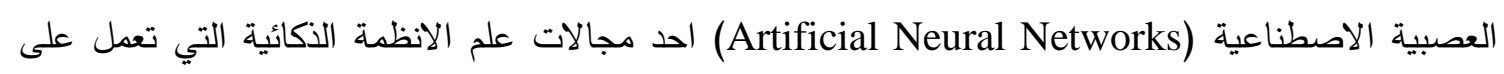
تمثيل عقل الانسان ونظامهُ المناعي من خلال شبكة من المعطيات الرقمية والتي يتم معالجتها بواسطة نماذج رياضية محددة [16]. إن الهدف من هذه الدراسة هو تقديم تقنية ذكائية مهنتة تعتمد على تقنيتي الثبكة المناعية

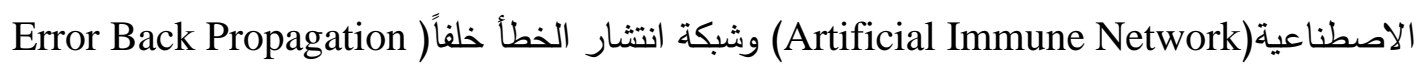
(Neural Network وتكون قادرة للتعرف على الانماط الطبية وتحديدا مرض وهن العظام. إذ تمت مقارنة نتائج

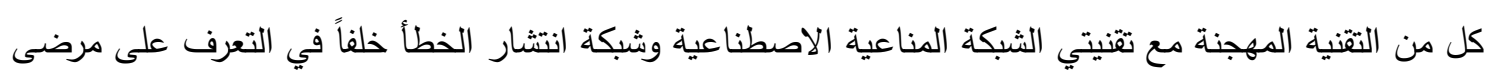

وهن العظام عن طريق المفاضلة بين هذه التقنيات من خلال الاعتماد على معيار متوسط معدل مربع الخطأ (Mean Square Error)

$$
\text { 2- 2 - 2 الدراسات السابقة }
$$

لقد أولى العديد من الباحثين اهتماما كبيرا لتحسين التقنيات الذكائية وأساليب تطويرها والاستفادة من الخصوصية الايجابية لكل تقنية والتغلب على بعض سلبيات العمليات الحسابية الناتجة عن هذه التقنيات, اذ قدم في العام (2005) كل من (Polat K. ,Sahan S.,Kodaz H. and Güneş S.) بحثاً وظفا فيه استخدام خوارزميات النظام المناعي لهني الاصطناعي مع المنطق المضبب في تصنيف الصور الرقمية, وفي العام (2007) قدم كل من الباحثين بحثاً تضمن استخدام الثبكة المناعية الاصطناعية في تتبؤ نتائج جينات من لوفئ (Tsankova D. and Rangelova V.) مرض السرطان وقد تضمنت الدراسة 58 عينة منها 32 من النوع القاتل و26 من النوع الذي يمكن شفاؤه.

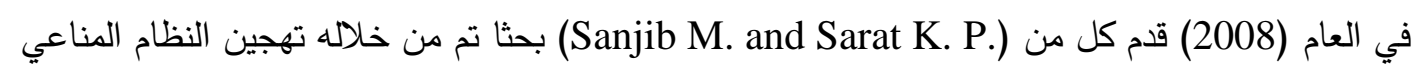
مع مجموعة من العمليات الجينية لغرض استخدامها في التتبؤ, كما تم تهجين النظام المناعي مع دالة الاساس الثعاعي وتطبيقها في السلاسل الزمنية من قبل كل من (Alexandrino J., Zanchettin C. and Filho E.) في العام (2009), وفي العام (2010) تمت دراسة السيطرة على المصعد من خلال النظام المناعي وطرق الامثلية

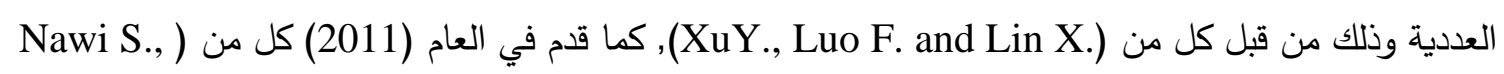
(Abdalla A. and Ramli M.

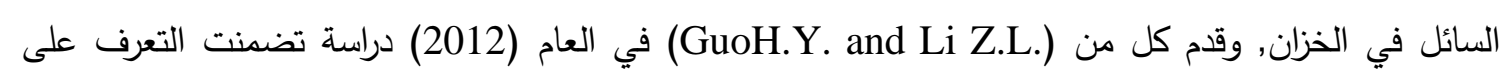
الأضرار الإنشائية من خلال نظرية بيز والخوارزمية المناعية.

\section{3-الأنظمة المناعية الاصطناعية والثبكات العصبية الاصطناعية}

تعد تقنية الأنظمة المناعية الاصطناعية (Artificial Immune Systems) والتي تكتب اختصارا (AIS)

وتقنية الشبكات العصبية الاصطناعية (Artificial Neural Network) والتي يطلق عليها اختصارا (ANN), من أهم أصناف علوم الحاسوب الحديثة في مجال علم الأنظمة الذكائية والتي يتم من خلالها محاكاة كل من نظام 
المناعة الطبيعي والنظام العصبي الطبيعي, وتعتمد كل تقنية من هذه التقنيات على مبادئ وعمليات مستوحاة من

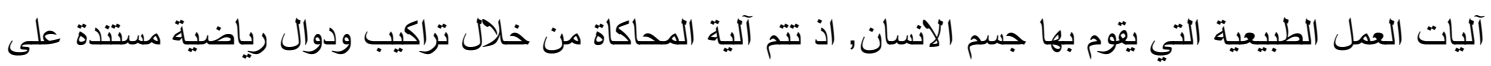
آلية المحاكاة لهذه الانظمة الحيوية [7][9] وقد حقق كل من الانظمة المناعية الاصطناعية والثبكات العصبية

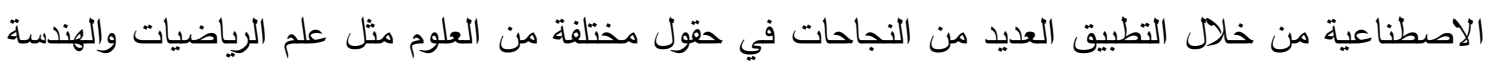

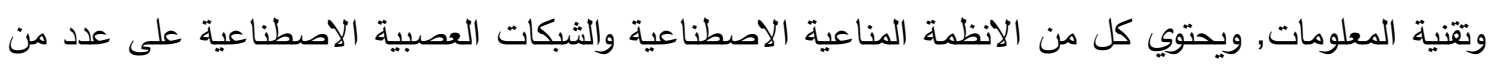

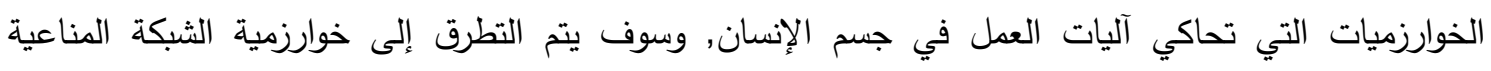
(AIS) مثال للانظمة المناعية الاصطناعية (Immune Network Algorithm) مثالاً للشبكات العصبية الاصطناعية (10) (ANNs (Back Propagation Neural Network)

Artificial Immune Network الثبكة المناعية الاصطناعية

تعد الشبكة المناعية الاصطناعية إحدى أهم خوارزميات الانظمة المناعية الاصطناعية والمستوحاة من الثبكة المناعية الطبيعية (Idiotypic) المقترحة من قبل العالم (Niels Kaj Jerne) في العام (1974)(4]), تعتمد في عملها على الخلايا المناعية نوع B-Cell المحفزة من قبل المستضد لتكون شبكة من الاجسام المضادة للتعرف على المستضد ويتم ذلك من خلال آليتين الاولى التحفيز (Activation) اذ يتم ربط مستقبل الجسم المضاد(ب) (Saratope) مع مستقبل المستضد (Epitope), أما الثانية الاخماد (Suppression) فيتم ربط مستقبل جسم مضاد (Adiotope) مع مستقبل جسم مضاد آخر (Idiotope(3][8]. أي إن قوة الربط تعتمد إلى حد كبير على درجة صلة الترابط

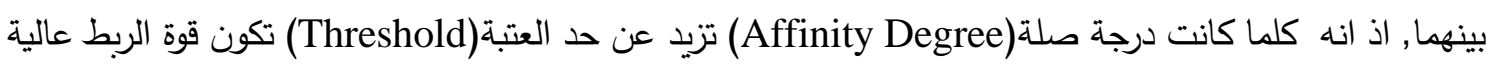

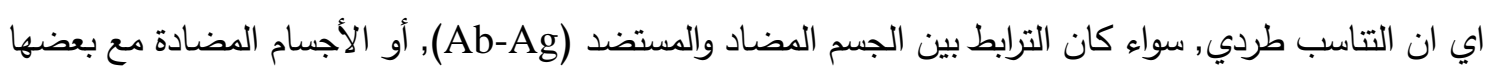
(Ab-Ab), ولها ميزة إدراكية مشابهة إلى حد ما آلية عمل الثبكات العصبية [12].

116] 13] موارزمية الثبكة المناعية الاصطناعية]

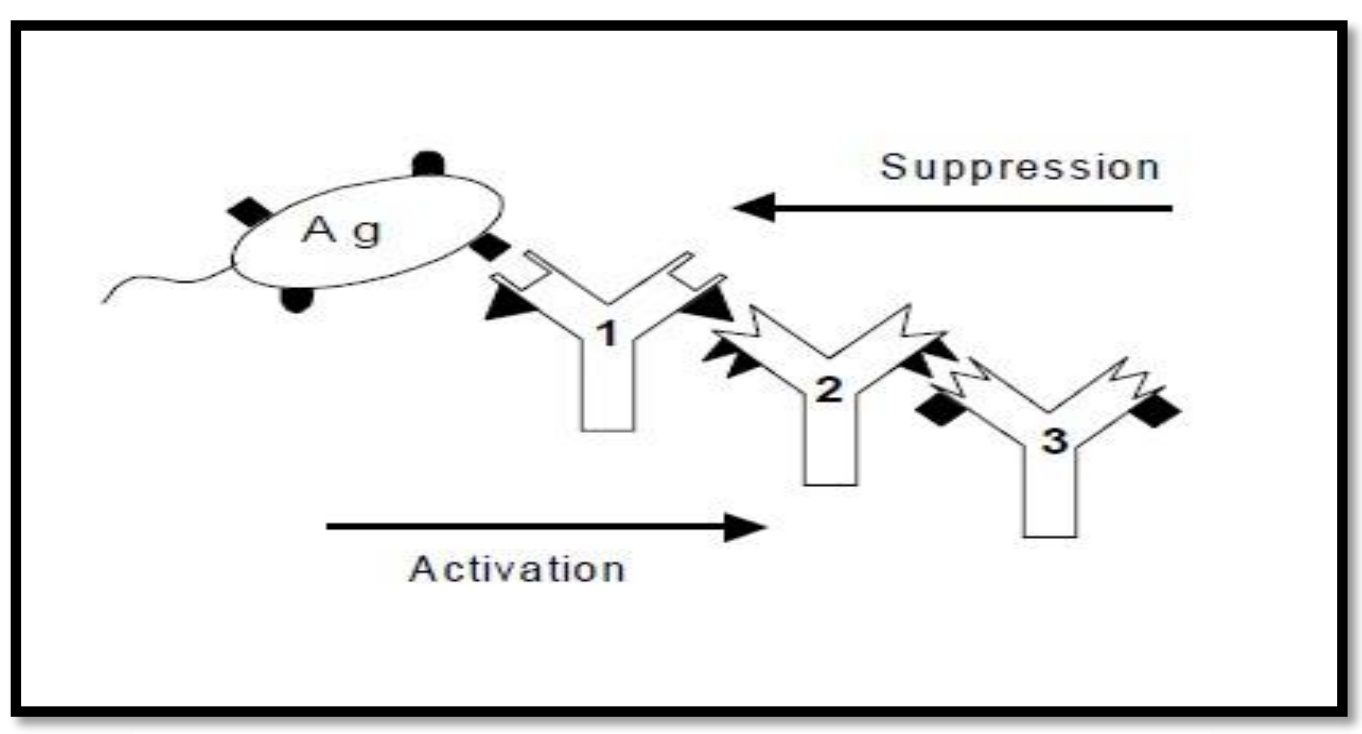

الثكل (1): يبين نموذج مبسط لآلية عمل الثبكة المناعية الطبيعية

الخطوة الاولى : التهيئة (Initialization) : إنثــــاء مجتمع ابتدائي عشــــائي من الأجســــام المضــــادة .(Antibody) 
الخطوة الثانية: التمثيل (Representation) : لكل مسـتضـــ (Antigen) الاسـتمرار في إجراء الخطوات

الانتقاء النسيلي (Clonal Selection) : لكل جسم مضاد يتم تحديد صلته (Affinity) مع المستضد

اختيار (nighest Affinity) من الخلايا ذات درجة الصلة العالية (ذ) يتم استتساخها بشكل يتتاسب مع صلتها ( أي التي تملك صلة عالية لها نسبة اختيار اكبر). تغيير (Mutate) كل جسم مضاد بصورة تتتاسب عكسيا مع درجة صلته.

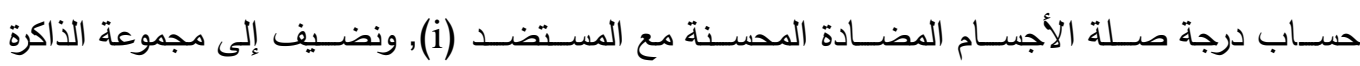
(Memory Set)

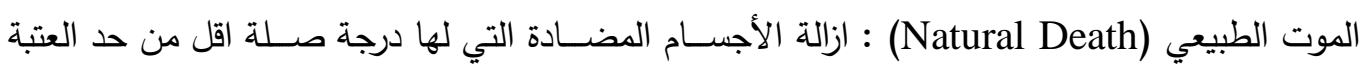
(Pruning Threshold) الإخماد النسيلي (Suppression Clonal) : حسـاب درجة الصـلة بين الأجسام المضــادة (Ab-Ab) : (Ab)

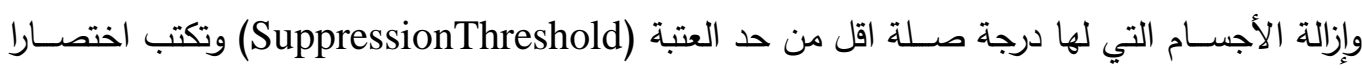

دمج الأجسام المضادة المتبقية من الذاكرة مع الأجسام المضادة للشبكة.

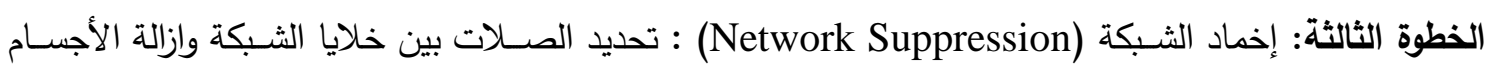
المضادة التي لها درجة صلة بالبقية التي تكون اقل من عتبة الإخماد (ts) .

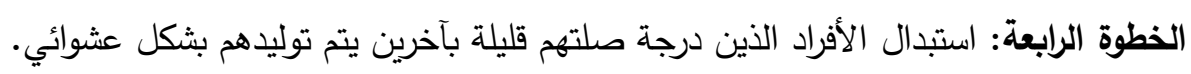
الخطوة الخامسة: اعادة الخطوات من (2-4) إلى أن يتحقق مقياس التوقف.

\section{5rror Back Propagation Neural Network شبكة الانتشار الخطأ خلفاً}

تعد هذه الثبكة من أكثر الثبكات العصبية الاصطناعية استخداماً والتي قد تبدو معقدة لكنها أسهل بكثير على مستوى الفهم والبرمجة ولها القدرة على التعامل مع المسائل غير الخطية وهي من الشبكات التيكة التي تتعلم بإشراف ولها العديد من التطبيقات في معالجة الصور ومعالجة الإثارة والتعرف على الكلام. طورت في منتصف 1970 من قبل (Rumelhart, Hinton and Williams) [13], أن هدف الثبكة هو تقليل الخطأ من خلال تعديل الأوزان (Weights) وذلك بمقارنة إخراج الثبكة الحقيقي (Actual Output) مع في

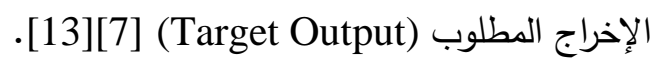
1-5 خوارزمية تعليم الاتتشار الخطأ خلفاً [11][13 و $W_{N}=\left(w_{1}, w_{2}, w_{3}\right.$ الخطوة الأولى : تهيئة الأوزان الابتدائية (1) - (Threshold)

$X_{N}=\left(\mathrm{x}_{1}, \mathrm{x}_{2}, \mathrm{x}_{3}, \ldots \ldots \ldots \mathrm{x}_{\mathrm{n}}\right)$ الخطوة الثانية: اختيار زوج التدريب (1) $Y_{\mathrm{J}}=\left(\mathrm{y}_{1}, \mathrm{y}_{2}, \mathrm{y}_{3}, \ldots \ldots . . . \mathrm{y}_{\mathrm{j}}\right)^{\mathrm{T}} \quad$ (Target Output) $\quad$ وتمثل 
الخطوة الثالثة : في الاتجاه الأمامي يتم حساب قيمة الإخراج الحقيقي (Actual Output) :

$n e t_{I H}=\left[\sum_{i=1}^{n} X_{I i} W_{i}-\theta_{i}\right]$ 1- حساب قيمة الإخراج الحقيقي من طبقة الإدخال إلى الطبقة المخفية

$Y_{H}=f\left(\right.$ net $\left._{I H}\right)$ n: تمثل عدد العناصر في طبقة الإدخال (Input Layer) للشبكة .

2- حساب قيمة الإخراج الحقيقي من الطبقة المخفية (Hidden Layer) إلى طبقة الإخراج Output)

$n e t_{H O}=\left[\sum_{j=1}^{P} Y_{H j} W_{j}-\theta_{j}\right]$

Layer)

P : تمثل عدد العناصر في الطبقة المخفية (Hidden Layer) للشبكة . P

$Y_{O}=f\left(\right.$ net $\left._{\mathrm{HO}}\right)$

$e_{j}=Y_{d}-Y_{j} \neq 0$

الخطوة الرابعة : حساب الخطأ ويتم من خلال الخطوات الآتية:

1- يتم تعديل الاوزان بين طبقة الإخراج (Output Layer) والطبقة المخفية (Hidden Layer)

$W_{j}^{\text {new }}=W_{j}^{\text {old }}+\Delta W_{j}$

$\Delta W_{j}=\alpha Y_{j} \delta_{j} \quad$ and $\delta_{j}=Y_{j}\left(1-Y_{j}\right) e_{j}$

2- يتم تعديل الاوزان بين الطبقة المخفية (Hidden Layer) وطبقة الإدخال (Input Layer)

$W_{\mathrm{i}}^{\text {new }}=\mathrm{W}_{\mathrm{i}}^{\text {old }}+\Delta \mathrm{W}_{\mathrm{i}}$

$\Delta \mathrm{W}_{\mathrm{i}}=\alpha \mathrm{X}_{\mathrm{i}} \delta_{\mathrm{i}}$ and $\delta_{\mathrm{i}}=\mathrm{Y}_{\mathrm{i}}\left(1-\mathrm{Y}_{\mathrm{i}}\right) \sum_{\mathrm{j}=1}^{\mathrm{p}} \delta_{\mathrm{j}} \mathrm{W}_{\mathrm{j}}$

الخطوة الخامسة: تكرار الخطوات من الخطوة الثانية إلى الخطوة الخامسة إلى أن نحصل على التقارب المطلوب (شرط التوقف).

6-وصف بيانات وهن العظام

تتضمن هذه البيانات عينات مختلفة من فحص مرض وهن العظام تتكون خلال مراحل مختلفة من الإصابة,

اذ تمثل حالة (Osteopenia) المرحلة الأولى من الإصابة بمرض وهن العظام, كما تمثل (Osteoporosis) المرحلة الثانية والمتأخرة من الإصابة بالمرض, في حين تكون الحالة الثالثة هي الحالة السليمة (Normal), ان البيانات التي تم استخدامها في التعرف على مرض وهن العظام عبارة عن قيم عددية ووصفية تمثل قاعدة أساسية لمعلومات ضمن قاعدة بيانات (Data Base) لأشخاص تم إجراء عملية الفحص عليهم داخل العراق وتحديداً في محافظة نينوى. تتكون مصفوفة البيانات التطبيقية من (344) نمطاً منها (113) نمطاً تشخيصها من نوع (Osteoporosis), في حين يوجد (131) نمطاً من نوع (Osteopenia), أما بقية الانماط والتي يبلغ عددها (100) نمطاً, يمثل

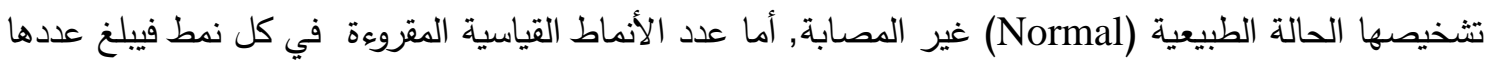

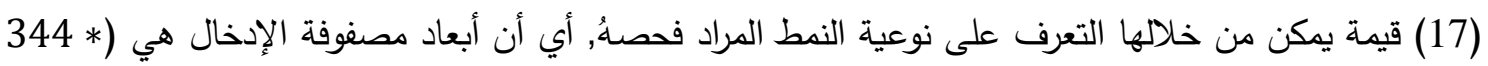

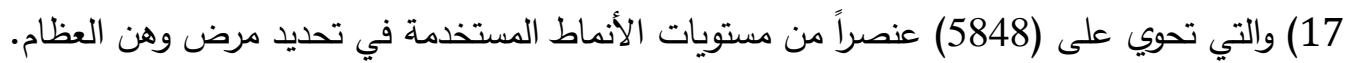




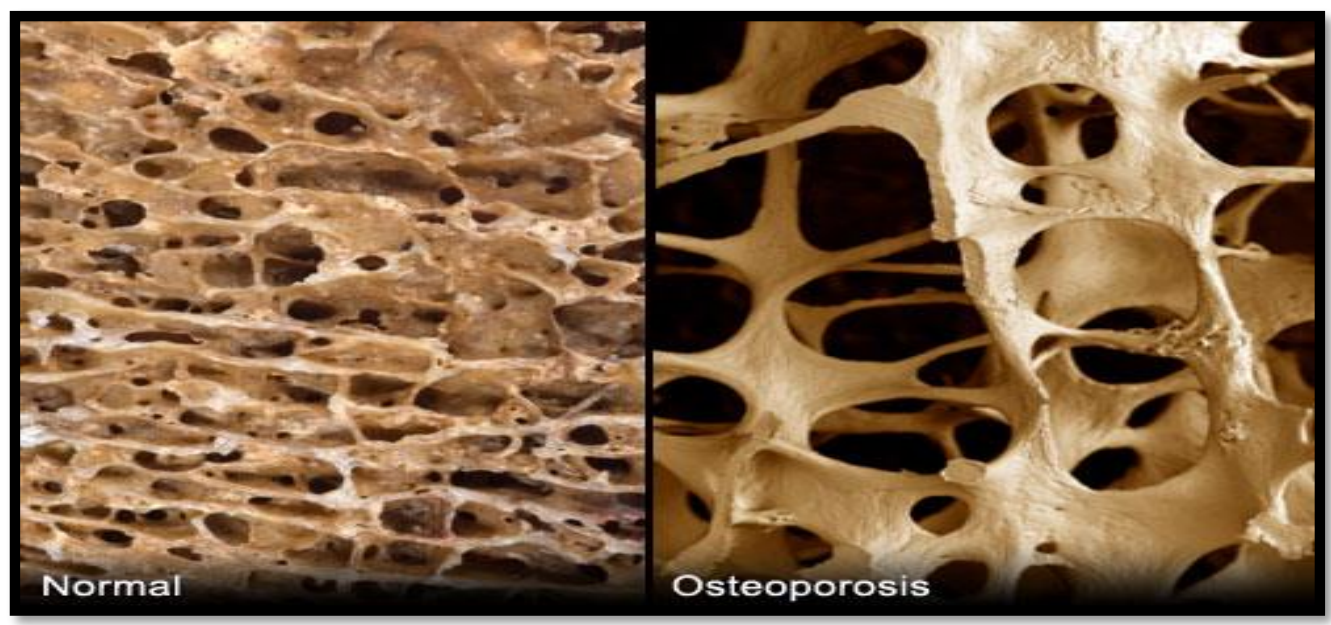

الثكلرقم(2): مقطع يوضح المقرنة بين النسيج الطبيعي (Normal)والنسيج المصاب بعرض

الوهن من فيع) الونسئ (Osteoporosis)

إن التثخيص المتقن والتعرف الدقيق على الأنماط يعد من أهم المسائل التي تحتاج إلى اختيار دقيق لتقنيات

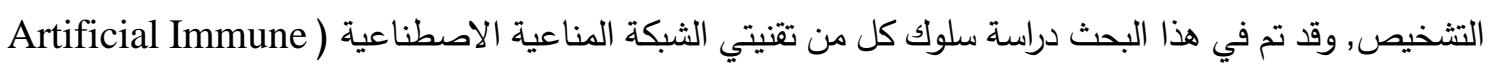
Network) والثبكة العصبية الاصطناعية (Artificial Neural Network), كما تم اقتراح خوارزمية مهجنة بين الثبكات العصبية الاصطناعية وتقنية الثبكات المناعية الاصطناعية من اجل التوصل إلى تقنية قادرة على التغلب على سلبيات كل من هاتين التقنيتين من خلال التركيز على ايجابياتها.

\section{Pattern Recognition System}

7- - 7 نظام التعرف على الأنماط

يعد التعرف على الأنماط أو تمييز الأنماط من تقنيات علم الأنظمة الذكائية, وهي من التقنيات المعروفة

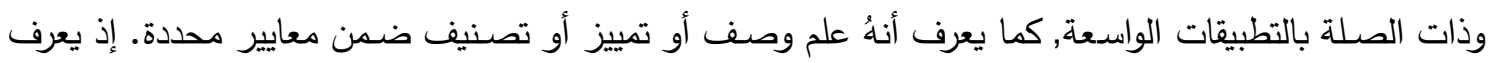

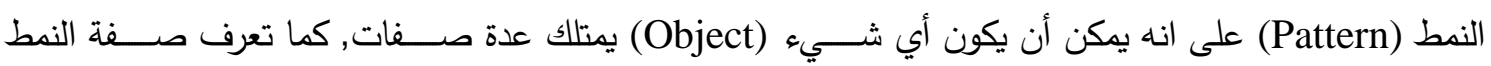
(Feature Pattern)

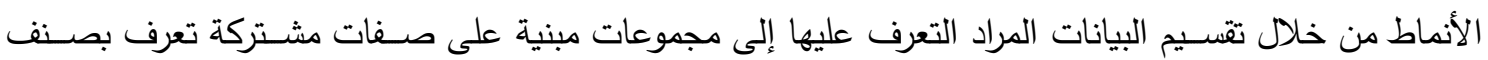

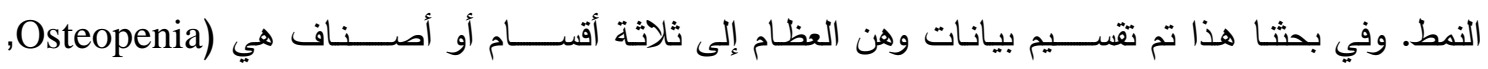

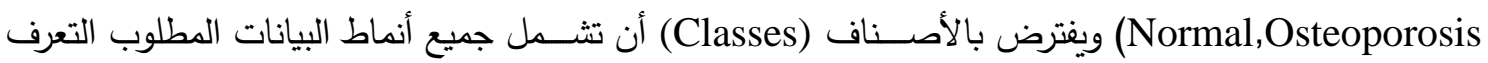
عليها, كما يتم التصـنيف هندسـيا من خلال إيجاد الحد الفاصـل (Decision Boundary) المحيط بكل منطقة تصنيف والذي قد يصعب إيجاده في بعض الأحيان بالأساليب الرياضية البسيطة المعروفة. 8- مقارنة الأظظة المناعية الاصطناعية والثبكات العصبية الاصطناعية

إن الآلية التي يعتمدها النظام المناعي الاصطناعي (AIS) في تكوين مصفوفة الذاكرة يعد أساسا مهما في

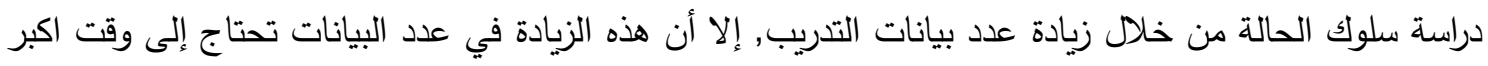

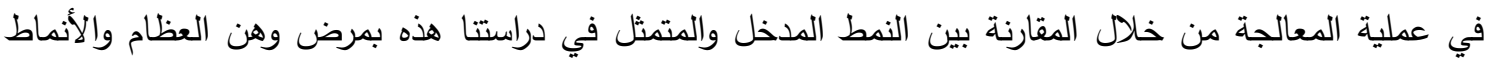
المخزونة في مصفوفة الذاكرة. كما أن تقنية الثبكات العصبية لها القدرة على معالجة البيانات من خلال تعديل قيم الاتصالات بين العقد التي تمثل أوزان الثبكة, إذ إن الآلية الرئيسية في الثبكات العصبية الاصطناعية هي الحصول 
على الأوزان المثالية, وهذه الآلية تتطلب بيانات تدريب عديدة وفي حالة عدم وجود بيانات كافية فان نتائج أنموذج الشبكة العصبية تكون غير دقيقة. لذلك فقد تم بناء نموذج مهجن قادر على التغلب على الصفات السلبية الموجودة في نظام المناعة والمتمثلة في الزمن الكبير نسبيا من خلال تمييز حالة مرضى وهن العظم قيد الدراسة وذلك من فن فئن

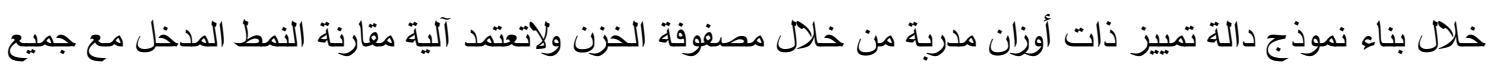
أنماط البيانات وإنما تعتمد على ضرب قيم النمط المدخل في الأوزان المقابلة له لإعطاء النتيجة وذلك من خلاد دلد دله المعادلة الآتية : - المانية

out $_{\mathrm{i}}=f\left(X_{i} * W_{i}\right)$

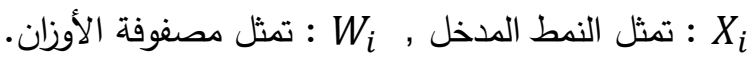

لألك فقد تم اخذ الصفة الايجابية التي يمتلكها النظام المناعي الاصطناعي وهي الذاكرة المناعية Memory

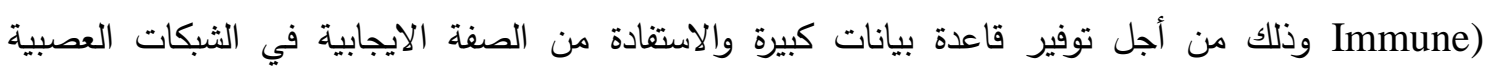
الاصطناعية من خلال الحصول على الأوزان المثالية.

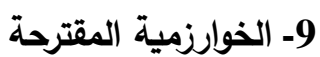

تتألف الخوارزمية المهجنة المقترحة من مرحلتين من مراحل معالجة أنماط البيانات, إذ يتم في المرحلة الأولى استخدام تقنية الثبكة المناعية الاصطناعية لأجل الحصول على أنماط بيانات كافية من خلال مفهوم مصفوفة الخزن والتي تحاكي الصفات الموجودة في أنماط بيانات الإدخال, في حين يتم تطبيق تقنية الثبكة

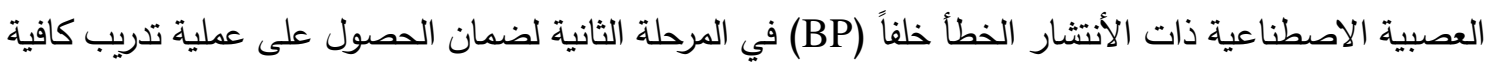
لتقليل نسبة الخطأ قدر الإمكان أثناء اختبار نماذج أنماط بيانات الإدخال, كما أن الخطوات التار التالية تبين آلية عمل

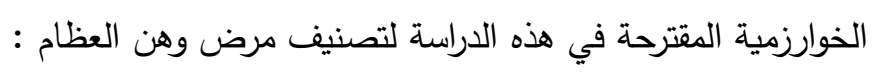
1- تهيئة أنماط بيانات الإدخال بشكل مصفوفة (X) بسعة, صفوفها تمثل عدد الأنماط المرضية والطبيعية وأعمدتها

$$
\text { تمثل عدد الأنماط التي تم قياسها في كل حالة. }
$$

2- تقسيم أنماط بيانات الإدخال إلى مجموعتين أساسيتين, تمثل الأولى بوصفها مجموعة أنماط بيانات التدريب

(X) (X) في حين تمثل المجموعة الثانية مجموعة أنماط بيانات الاختبار ( بواقع ثلث حالات مجموعة أنماط البيانات الكلية. 3- استخدام مجموعة أنماط بيانات التدريب (Xraining) في بناء مصفوفة الخزن (Memory Matrix) وذلك من خلال تطبيق مفاهيم الشبكة المناعية الاصطناعية. 4- استخدام مصفوفة الخزن (Memory Matrix) الناتجة من الثبكة المناعية كمجموعة أنماط تدريب في بناء وملاءمة نموذج الثبكة العصبية الاصطناعية لتكوين الأوزان المثالية. 5- بناء تقنية نهائيه يستخدم بوصفه دالة تصنيف للحالات المرضية, وذلك بالاعتماد على الأوزان المثالية الناتجة من الثبكة المدربة. 6- مقارنة نتائج مجموعة أنماط بيانات الاختبار ( Xest ) مع النتائج الحقيقية, بعد تطبيقها على النموذج النهائي لمعرفة مدى كفاءته. 


\section{1-9 تطبيق الخوارزمية المقترحة على بيانات وهن العظام}

تعد الخوارزميات المقترحة نموذجا حاسوبيا يعتمد مبادئ علم الانظمة الذكائية وذلك من خلال بناء أنظمة قادرة

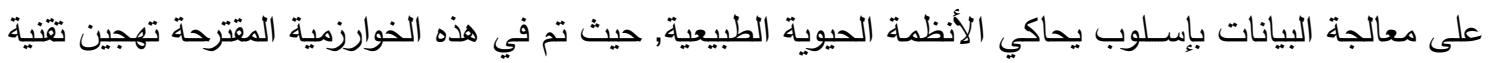
الثبكة المناعية الاصـناعية وذلك من خلال تقنية الثبكة العصبية الاصطناعية, من اجل الحصـول على تقنية

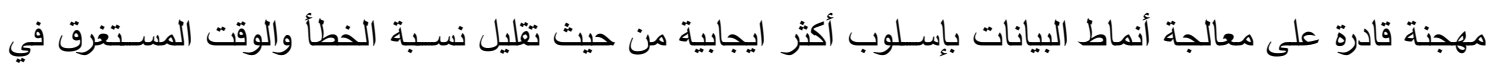

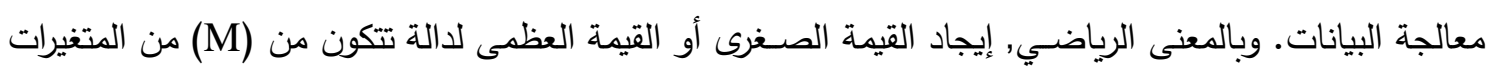
. $f\left(X_{1}, X_{2}, \ldots, X_{M}\right)$

لقد تم تطبيق الخوارزمية المهجنة المقترحة على بيانات وهن العظام ومقارنتها مع كل من الثبكة المناعية

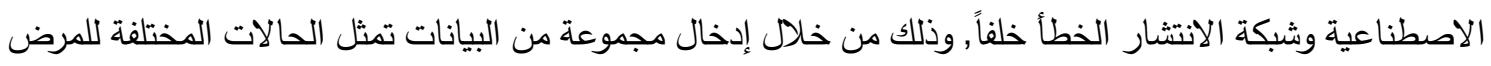
وهي (Osteoporosis, Osteopenia, Normal), حيث إن كل نمط يتكون من مجموعة من القيم عددها (17) قيمة تمثل قراءات يتم من خلالها التعرف على أنماط الحالة واهم هذه القراءات هي (T-score, Z-score) وهما

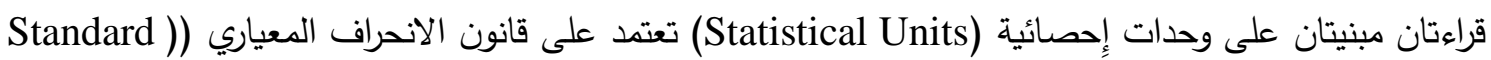

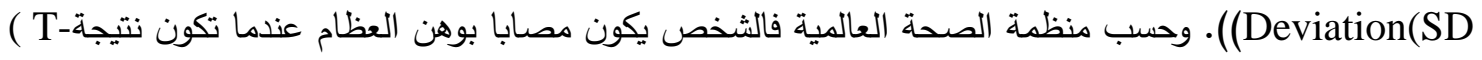
أقل من (2.5-80re )

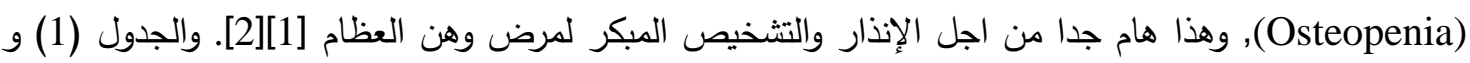

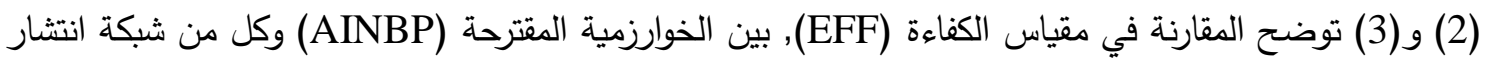
الخطأ خلفاً (BP) والثبكة المناعية الاصطناعية (AIN). وتم الاعتماد على عدد من المقاييس منها مقياس الزمن

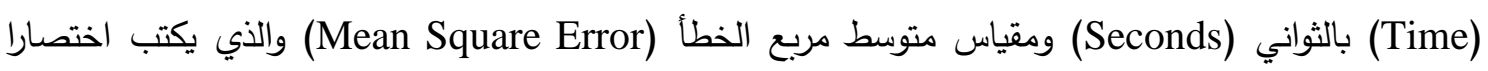
$M S E=\frac{1}{N} \sum_{i=1}^{N}\left(t_{i}-\text { Actual }_{i}\right)^{2}$

$$
\text { (MSE) }
$$

t t : بمثل الإخراج المطلوب (Target Output ) لبيانات وهن العظام. ئرثل الإخراج الحقيقي (Actual

كما تم حسـاب مقياس الكفاءة (Efficiency Scale) والذي يكتب اختصـارا (EFF) ويمثل الإجنه المحصـلة النهائية لكل

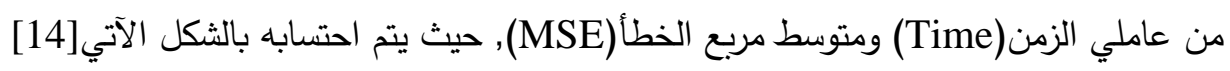
$E F F=1 /(M S E *$ Time $)$

الجدول (1) : مقارنة بين شبكة (BP) والخوارزمية المقترحة في التعرف على حالات مرضى وهن العظام.

\begin{tabular}{|c|c|c|c|}
\hline $\begin{array}{c}\text { مقياس الكفاءة } \\
\text { EFF }\end{array}$ & الزين بالثانية & معدل مربع الخطأ MSE & $\begin{array}{c}\text { نوع التقنية } \\
\text { Type of Technique }\end{array}$ \\
\hline 21.1149 & 6.4 & 0.0074 & $\begin{array}{c}\text { (AINBP) } \\
\text { (الخوارمية المقترحة }\end{array}$ \\
\hline 1.7337 & 1.6 & 0.3605 & الثبكة العصبية الاصطناعية \\
\hline
\end{tabular}


الجدول (2) : مقارنة بين شبكة (AIN) والخوارزمية المقترحة في التعرف على حالات مرضى وهن العظام.

\begin{tabular}{|c|c|c|c|}
\hline $\begin{array}{l}\text { مقياس الكفاءة } \\
\text { EFF }\end{array}$ & الزمن بالثانية & معدل مربع الخطأ & $\begin{array}{c}\text { نوع التقنية } \\
\text { Type of Technique }\end{array}$ \\
\hline 21.1149 & 6.4 & 0.0074 & $\begin{array}{c}\text { الخوارزمية المقترحة } \\
\text { (AINBP) }\end{array}$ \\
\hline 0.0460 & 24.5 & 0.8870 & الثبكة المناعية الاصطناعية \\
\hline
\end{tabular}

نلاحظ من الجدولين (1) و (2) أن الطريقة المهجنة المقترحة (AINBP) تعطي نتيجة أفضل من الثبكة

العصبية الاصطناعية (BP) وأفضل من الثبكة المناعية الاصطناعية (AIN) في تصنيف بيانات مرض وهن العظام, وذلك من خلال مقياس معدل مربع الخطأ (MSE), في حين نلاحظ أن الزمن المستغرق في معالجة البيانات باستخدام التقنية المهنة المقترحة (AINBP) هو أقل من الزمن المستغرق في معالجة البيانات باستخدام الثبكة المناعية الاصطناعية (AIN) واكبر من الزمن المستغرق في معالجة البيانات باستخدام الشبكة العصبية الاصطناعية (BP), وبوجود الاختلاف في كل من عاملي الزمن ونسبة الخطأ يمكن الاستعانة بمقياس الكفاءة (EFF) والذي الذي الذيات يمثل النتيجة المستخلصة من عاملي الزمن وقياس متوسط مربع الخطأ (MSE) ويمكن الاعتماد عليه بوصفه نتيجة نهائية, اذ اثبت التقنية المهنة المقترحة (AINBP) هي الأفضل من كل من تتنيتي شبكة انتشار الخطأ خلفاً (BP)

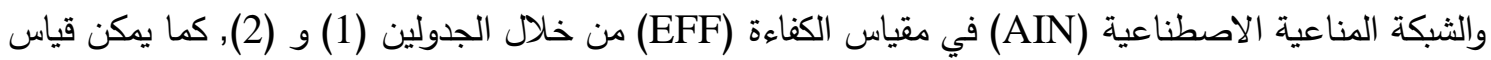
النسبة المئوية لكفاءة الخوارزمية المقترحة (AINBP) بالنسبة لتقنيتي كل من شبكة الأنتشار الخطأ خلفاً (BP) $I(B P)_{E F F}=\frac{E F F(\mathrm{BP})}{E F F(\mathrm{AINBP})} * 100 \%=8.2108 \%$ والشبكة المناعية الاصطناعية (AIN) كما يأتي :

$I(A I N)_{E F F}=\frac{E F F(A I N)}{E F F(\mathrm{AINBP})} * 100 \%=0.2179 \%$

أي أن كفاءة النسبة المئوية لتقنية شبكة انتشار الخطأ خلفا (BP) مقارنة بالطريقة المقترحة (AINBP) تعادل

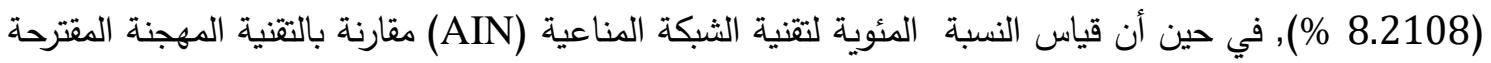
(AINBP) الجدول (3) : مقارنة بين التقنية المقترحة (المهجنة AINBP) وكل من شبكة (AIN) وشبكة (BP) في التعرف على حالات مرضى وهن العظام من حيث مقياس الكفأوة .

\begin{tabular}{|c|c|c|}
\hline تصنيفها بالات الاختبار التي تمل صيح & مقياس الكفاءة (EFF) & $\begin{array}{c}\text { Type of ( نوع التقنية المستخدمة } \\
\text { (Technique }\end{array}$ \\
\hline $69 / 115$ & 1.7337 & الثبكة العصبية الاصطناعية (BP) \\
\hline $13 / 115$ & 0.0460 & الثبكة المناعية الاصطناعية (AIN) \\
\hline $115 / 115$ & 21.1149 & الخوارزمية المقترحة (AINBP) \\
\hline
\end{tabular}


نلاحظ من الجدول (3) أن التقنية المهجنة المقترحة (AINBP) تعطي نتيجة أفضـل في تصـنيف بيانات

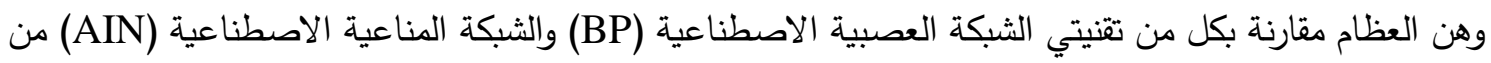
خلال مقياس الكفاءة (EFF), كما نلاحظ أن الكفاءة النســبية للزيادة في التقنية المهجنة المقترحة (BINBP)

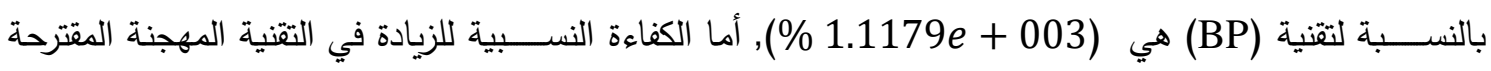

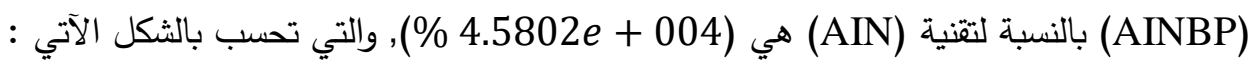

$R 1_{E F F}=\frac{E F F(A I N B P)-E F F(\mathrm{BP})}{E F F(\mathrm{BP})} * 100 \%$

$R 2_{E F F}=\frac{E F F(A I N B P)-E F F(\mathrm{AIN})}{E F F(\mathrm{AIN})} * 100 \%$

10-الاستنتاجات والتوصيات

تضمنت هذه الدراسة خوارزمية مقترحة (مهجنة) بين تقنيتي كل من الثبكة المناعية الاصطناعية (AIN) وشبكة انتشار الخطأ خلفا (BP) واستخدامها في التعرف على مرضى وهن العظام. ومن خلال التطبيق العملي تبين

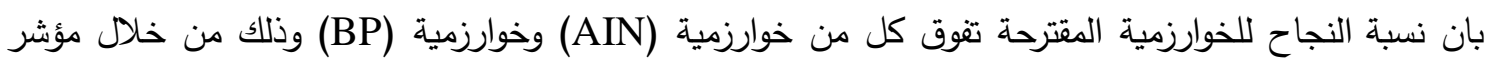

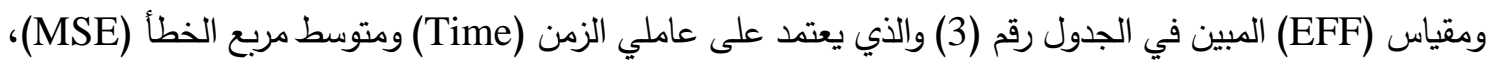
مما يدل على الكفاءة العالية لهذه الخوارزمية. وفي ختام هذه الدراسة نوصي بالتوسع بدراسة التتنيات الذكائية وعمليات

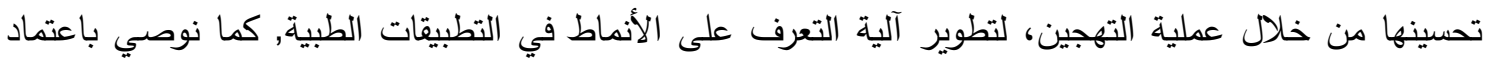
الطريقة المقترحة في المراكز الطبية المختصة في مرض وهن العظام لمساعدة الأطباء في تحديد نوعية المرض. 
المصادر

[1] مركز التمييز لأبحاث وهن العظام, جامعة الملك عبد العزيز - جميع الحقوق محفوظة لعمادة تقنية المعلومات

(2012), على الرابط: Www.kau.edu.sa.

[2] ويكيبيديا الموسوعة الحرة،(2012), على الرابط: http://en.wikipedia.org/wiki/Osteoporosis.

[3] Aanchal Malhotra, Abhishek Baheti, Shilpi Gupta,(2012),"Pattern

Recognition Approaches inspired by Artificial Immune System"

International Journal of Computer Applications (0975 - 8887) Volume 44 No20,pp(12-16).

[4] Al-Enezi J.R.,Abbod M.F., and Alsharhan S.,(2010), "Artificial Immune Systems-Models, Algorithms and Applications", IJRRAS3 (2) , pp(118131).

[5] Bhagat Phiroz,(2005), " Pattern Recognition in Industry". International Strategy Engines New Jersey, USA.

[6] Bishop Christopher M.,(2006),"Pattern Recognition and Machine Learning", Springer .

[7] Ertel Wolfgang,(2011)," Introduction to Artificial Intelligence", Translated by Nathanael Black With illustrations by Florian Mast, Springer-Verlag London Limited 2011, www.springer.com/series/7592.

[8] Gu Feng,(2011),"Theoretical and Empipirical Extensions of The Dendritic Cell Algorithm", Thesis submitted to the University of Nottingham for the degree of Doctor of Philosophy .

[9] Gunasekaran M., Ramaswami K.S.,(2011," Evaluation of Artificial Immune System with Artificial Neural Network for Predicting Bombay Stock Exchange Trends", Journal of Computer Science 7 (7): pp967972,ISSN 1549-3636.

[10] Khonde R. D. , Pandharipande S. L.,(2011), " Application of Artificial Neural Network for Standardization of Digital Colorimeter", International Journal of Computer Applications (0975 - 8887),pp(1-4).

[11] Negnevitsky Michael,(2005),"Artificial Intelligence A Guide to Intelligent Systems", Second Edition, ISBN 0321204662.

[12] Shrivastava Amit Kumar,(2011)," Optimization of Robotic Assembly of Printed Circuit Board Using Volutionary Algorithm ", Master of Technology in Machine Design and Analysis.

[13] Sumathi S.,Surekha P.,(2010),"Computational Intelligence Paradigms Theory and Applications using MATLAB",CRC Press is an imprint of the Taylor \& Francis Group, an inform business. 
[14] Steel R.G.D. and Torrie J.H., (1980), "Principles and Procedures of Statistics a Biometrical Approach", Mc Graw-Hill, Inc.

[15] The Odoridis Sergios,(2003),"Pattern Recognition", Second Edition.

[16] Yang Liu,(2009),"A Neuro-Immune Inspired Computational Framework and Its Application to A Machine Visual Tracking System", PH.D., The university of York department of Electronics. 